\title{
Clinical performance of the Nova $\mathrm{T380}^{\circledR}$ intrauterine device in routine use by the UK Family Planning and Reproductive Health Research Network*: 5-year report
}

\author{
Michael Cox, FRCOG, MFFP, Consultant Obstetrician and Gynaecologist (Retired), Nuneaton, UK; John Tripp, MD, FRCPCH, \\ School of Postgraduate Medicine and Health Sciences, University of Exeter, Exeter, UK; Sarah Blacksell, BA, Research Fellow, \\ School of Postgraduate Medicine and Health Sciences, University of Exeter, Exeter, UK
}

Correspondence: UK Family Planning and Reproductive Health Research Network, c/o John Tripp, Department of Child Health, Church Lane, Heavitree, Exeter EX2 5SQ, UK

\begin{abstract}
*Principal investigators: V Annandale (Norwich), A Barnett (Exeter), P Barnes (Richmond, London), J Bateman (Portsmouth), J Bland (Nuneaton), D Booker (Newport), R Bradbury (Barnsley), S Brown (Birmingham), G Cardy (Bristol), S Carr (Glasgow), $H$ Cooling (Bristol), M Cox (Nuneaton), J Dewsbury (Birmingham), J Elstub (Richmond, London), S Green (Cumbria), B Hanson (Wiltshire), T Laverty (Wiltshire), A Main (Richmond, London), H Massil (London CHSL), C Nash (Norwich), R Owen (Taunton), S Randall (Portsmouth), S Richardson (West Yorkshire), S Rowlands (Biggleswade), J Rumsey (Birmingham), $J$ Tattersall (Cumbria), A Taylor (Sheffield), A Thomas (Newport), C Tupper (Cumbria), A Turner (Wiltshire), $C$ Watson (London CHSL), E M Watt (Bristol)
\end{abstract}

(Accepted $26^{\text {th }}$ February 2002)

The Journal of Family Planning and Reproductive Health Care 2002: 28(2): 69-72

\begin{abstract}
Objectives. The purpose of the study was to evaluate the pregnancy and complication rates of this new device, with its increased area of copper, in comparison with other published results, in the clinical setting of British general practice and family planning clinics.

Design. Doctors working in general practice and at family planning clinics throughout the UK who collaborate in the UK Family Planning and Reproductive Health Research Network were responsible for the fitting of 574 Nova T380 ${ }^{\circledR}$ intrauterine contraceptive devices (IUDs). The Nova $T^{\circledR}$ (and formerly the identical Novagard ${ }^{\circledR}$ ) IUDs have copper with a surface area of $200 \mathrm{~mm}^{2}$. The Nova T380 ${ }^{\circledR}$ has copper with a surface area of $380 \mathrm{~mm}^{2}$.

Results. This is the first 5-year report on this device. The 5-year cumulative life-table event rates per 100 women were pregnancy 2.0, expulsion 13.0, and removal for bleeding problems and bleeding with pain 29.6.

Conclusions. The increased surface area of copper was associated with a reduced pregnancy rate as compared to the Nova $T^{\circledR}$, though no statistical comparison is possible. Although the present study was not a direct comparative study with the Nova $T^{\circledR}$, the result lends weight to the notion that increasing the copper reduces the pregnancy rate. The discontinuation rate for bleeding problems and bleeding with pain and the expulsion rates were higher than in published Nova $T^{\circledR}$ studies.
\end{abstract}

\section{Key message points}

- At 5 years of use in the UK the Nova $\mathrm{T} 380^{\circledR}$ intrauterine device (IUD) has been shown to have a very low pregnancy rate.

- There was a higher than expected removal rate for bleeding/pain.

- There was a higher than expected expulsion rate.

- There was a very low removal rate for pelvic inflammatory disease (PID).

- The study shows that this device is a welcome addition to the copperbearing IUDs available.

\section{Introduction}

This report concludes the study of which the 12-month and 24-month results have been published., ${ }^{1,2}$ It has been suggested that the contraceptive efficacy of copper intrauterine devices (IUDs) correlates positively to the area of copper-releasing surface but the evidence has been conflicting. ${ }^{3-6}$ Nevertheless it is now generally thought that increased copper does reduce pregnancy rates and prolong usability of the device. ${ }^{7}$ The Nova $\mathrm{T}^{\circledR}$ IUD (also available as the Novagard ${ }^{\circledR}$ until May 1998) has a copper wire on the central stem with a surface area of $200 \mathrm{~mm}^{2}$. The present study is of the Nova T380 ${ }^{\circledR}$ in which the surface area of copper has been increased to $380 \mathrm{~mm}^{2}$, all the copper being on the central stem. The purpose of the present study was to evaluate the pregnancy and complication rates of this new device, with its increased area of copper, in comparison with other published results, in the clinical setting of British general practice and family planning clinics.

\section{Methods}

The study was an open, single group, multicentre phase III study in outpatients. Doctors working in general practice and at family planning clinics throughout the UK who collaborate in the UK Family Planning and Reproductive Health Research Network were responsible for the Nova $\mathrm{T} 380^{\circledR}$ fittings reported in this paper. The study was sponsored by Leiras Oy and Schering Health Care Limited. Ethics committee approval for the study was obtained at all centres.

The Nova $\mathrm{T} 380^{\circledR}$ has a polyethylene frame shaped as a modified T with $380 \mathrm{~mm}^{2}$ of silver-cored copper wound around the vertical arm. The silver core is intended to prevent corrosion-induced fragmentation.

The device was fitted according to the doctor's own clinical judgement, in parous women attending for family planning advice and requesting intrauterine contraception. Those enrolled were aged $18-45$ years, exposed to the risk of pregnancy, in good health and with normal menstrual 
cycles. They were fitted with their IUD more than 6 weeks after pregnancy ended. After entry to the study, the subjects were followed for 5 years.

After insertion of the IUD, each subject was requested to return for follow-up examination at 3 months, 12 months and annually thereafter. Baseline data and details of the fitting procedure and follow-up visits were forwarded to the study co-ordinator at the University of Exeter, where the data were processed and analysed. Event rates were calculated using the life-table method. ${ }^{8}$ Adverse events were classified using the WHO Adverse Reaction Dictionary (Version 1/1995).

A programme of comprehensive monitoring using EU Good Clinical Practice (GCP) Guidelines was used. At the close of recruitment 574 women had been recruited who satisfied the criteria of the study protocol (reasons for protocol violation are listed in Table 1). This paper reports the data from this per protocol sample for 5 years of use. These data have been separately analysed on an intentionto-treat (ITT) basis by the sponsor. ${ }^{9}$ No rates vary to any clinically or statistically significant degree from those given. Some minor adjustments to the data and the sample occurred as a result of monitoring during the later stages of the study and also the use of additional methods of contraception by some subjects during the course of the study (seven removals from the sample, nine reinstatements). The use of hormonal methods of contraception was a protocol violation for this study. As a result the sample differs slightly from the sample used in the previous papers on 12 and 24 months of use. ${ }^{1,2}$

Table 1 Reasons for protocol violation $(n=83)$

\begin{tabular}{lr}
\hline Reason & $\mathrm{n}$ \\
\hline Over 45 years of age & 16 \\
Nulliparous & 9 \\
Second or subsequent fitting & 2 \\
IUD fitted as emergency contraception & 2 \\
Pregnant at fitting & 5 \\
Fitting at less than 6 weeks since live birth & \\
Concomitant method; using POP for more than one cycle & \\
Concomitant method; using COC or HRT & \\
Concomitant method; subject using other methods (pessaries, \\
spermicides)
\end{tabular}

COC, Combined oral contraceptive; HRT, hormone replacement therapy; POP, progestogen-only pill.

\section{Results}

\section{Demographic characteristics}

The mean age of the study population was 33 years (range 18-45 years). All the women were parous. The age distribution is shown in Table 2.

Table 2 Age distribution

\begin{tabular}{llr}
\hline Age group (years) & $\mathrm{n}$ & $\%$ \\
\hline $18-24$ & 53 & 9.2 \\
$25-29$ & 157 & 27.4 \\
$30-34$ & 140 & 24.4 \\
$35-39$ & 149 & 26.0 \\
$40-45$ & 75 & 13.0 \\
Total & 574 & 100 \\
\hline
\end{tabular}

\section{Cumulative life-table gross closure rates}

The cumulative gross rates per 100 women for closure of study at 12, 24, 36, 48 and 60 months are given in Table 3. At 5 years of use, the pregnancy rate was very low [2.0, $95 \%$ confidence interval (CI) 0.9-4.0]. Expulsion rates were higher than expected $(13.0,95 \%$ CI 9.5-16.4) at 5 years of use. The rate for removal for bleeding problems and bleeding with pain was also high $(29.6$, 95\% CI 24.7-34.5) at 5 years. The main closure rates, from other studies, for the Nova $\mathrm{T}^{\circledR}$ and the Gyne-T380 ${ }^{\circledR}$ at 5 years and the rates for the present study are given in Table 4 .

\section{Pregnancy}

The cumulative gross rate per 100 women for pregnancy was 2.0 (95\% CI 0.9-3.9) at 5 years. There were eight pregnancies, all occurring during the first 3 years, of which three were terminated, two were ectopic, two were spontaneous abortions and one was a normal delivery. Two other women who were excluded as protocol violators [one fitted less than 6 weeks after last live birth, one taking concomitant combined oral contraceptives (COC)] became pregnant. Analysing the data on an ITT basis, which includes these two women, gives a 60 -month pregnancy rate of 2.82 (95\% CI $0.91-4.74)$. Four pregnancies occurred during the first 12 months of the study and were described in the 12 -month report. ${ }^{1}$ The remaining four pregnancies are described below. It is notable that of the eight cases, four were associated with partial expulsion and one with complete expulsion of the IUD.

Case report 1. Pregnancy diagnosed 14 months after insertion. Pregnancy terminated and the IUD removed. Duration of pregnancy 10 weeks.

Case report 2. Pregnancy diagnosed 14 months after insertion when the IUD was found to be partially expelled. Pregnancy continued with a normal delivery at 39 weeks of a healthy baby.

Case report 3. Pregnancy diagnosed 13 months after insertion. The IUD was found to be partially expelled and was removed. The patient wished to continue the pregnancy but had a spontaneous abortion the next day. Duration of pregnancy 5 weeks.

Case report 4 . Twenty-nine months after insertion the threads could not be found. The patient was advised to use condoms pending ultrasound scan. Period was a week late at time of scan, which showed no IUD. Pregnancy diagnosed. The patient wished to continue with the pregnancy but had a spontaneous abortion in first trimester; exact duration of pregnancy not known. It is assumed there had been an unnoticed expulsion.

IUD removal following a complaint of bleeding and bleeding with pain

A total of 103 women had the device removed at or before 5 years following a complaint of bleeding problems or of bleeding with pain, giving a rate of 29.6 at 5 years (Table 3 ). The average length of use before removal of the device for these reasons was 19.4 months. The types of bleeding problems are shown in Table 5. Data on methods of treating bleeding or pain was not collected systematically.

Other medical complaints requiring IUD removal

A total of 13 women had the device removed at or before 5 years following medical complaints associated with the IUD giving a rate of 4.3 (Table 3). Male dyspareunia was a contributory factor in four cases. Other complaints included stomach cramps, backache, Candida infection, vaginal irritation, recurrent urinary tract infection (UTI), water retention, and offensive vaginal discharge.

Pelvic inflammatory disease (PID)

The diagnosis depended on the judgement of the clinician involved. There were ten cases of PID (Table 6). One case 
Table 3 Cumulative life-table gross closure rates per 100 users at 12, 24, 36, 48 and 60 months and net continuation (per protocol sample of 574)

\begin{tabular}{|c|c|c|c|c|c|c|c|c|c|c|c|c|c|c|c|}
\hline & \multicolumn{3}{|c|}{12 months } & \multicolumn{3}{|c|}{24 months } & \multicolumn{3}{|c|}{36 months } & \multicolumn{3}{|c|}{48 months } & \multicolumn{3}{|c|}{60 months } \\
\hline & $\mathrm{n}$ & GR & $\mathrm{CI}$ & $\mathrm{n}$ & GR & $\mathrm{CI}$ & $\mathrm{n}$ & GR & $\mathrm{CI}$ & $\mathrm{n}$ & GR & $\mathrm{CI}$ & $\mathrm{n}$ & GR & $\mathrm{CI}$ \\
\hline Pregnancy & 4 & 0.8 & $0.2-2.0$ & 7 & 1.6 & $0.7-3.4$ & 8 & 2.0 & $0.9-4.0$ & 8 & 2.0 & $0.9-4.0$ & 8 & 2.0 & $0.9-4.0$ \\
\hline Expulsion & 31 & 6.0 & $3.9-8.1$ & 40 & 8.6 & $6.0-11.2$ & 45 & 10.3 & $7.4-13.2$ & 49 & 12.3 & $9.0-15.6$ & 50 & 13.0 & $9.5-16.4$ \\
\hline \multicolumn{16}{|l|}{ Side effects } \\
\hline Perforation & 0 & 0.0 & $0.0-0.0$ & 0 & 0.0 & $0.0-0.0$ & 0 & 0.0 & $0.0-0.0$ & 0 & 0.0 & $0.0-0.0$ & 0 & 0.0 & $0.0-0.0$ \\
\hline Bleeding problems & 48 & 10.3 & $7.5-13.1$ & 70 & 16.2 & $12.6-19.7$ & 84 & 21.1 & $17.0-25.1$ & 97 & 26.5 & $21.9-31.1$ & 103 & 29.6 & $24.7-34.5$ \\
\hline Pain & 9 & 1.9 & $0.9-3.6$ & 14 & 3.4 & $1.6-5.2$ & 17 & 4.5 & $2.4-6.7$ & 19 & 5.5 & $3.0-7.9$ & 21 & 7.1 & $4.1-10.1$ \\
\hline PID & 4 & 0.9 & $0.2-2.3$ & 4 & 0.9 & $0.2-2.3$ & 4 & 0.9 & $0.2-2.3$ & 4 & 0.9 & $0.2-2.3$ & 4 & 0.9 & $0.2-2.3$ \\
\hline \multicolumn{16}{|l|}{$\begin{array}{l}\text { Other complaint } \\
\text { associated with }\end{array}$} \\
\hline IUD use & 7 & 1.6 & $0.6-3.2$ & 9 & 2.2 & $1.0-4.1$ & 11 & 3.0 & $1.2-4.8$ & 12 & 3.6 & $1.6-5.6$ & 13 & 4.3 & $2.0-6.6$ \\
\hline \multicolumn{16}{|l|}{ Not related } \\
\hline Planning pregnancy & 17 & 4.1 & $2.1-6.0$ & 29 & 7.5 & $4.8-10.2$ & 45 & 13.3 & $9.6-17.0$ & 52 & 16.5 & $12.3-20.7$ & 57 & 19.5 & $14.8-24.1$ \\
\hline $\begin{array}{l}\text { Medical not related } \\
\text { to IUD }\end{array}$ & 1 & ( 3 & $00-15$ & 4 & 13 & $03-32$ & 5 & 16 & $05-38$ & 5 & 16 & $05-38$ & 5 & 16 & $0.5-3.8$ \\
\hline Other reason & 16 & 3.6 & $1.8-5.4$ & 30 & 7.8 & $5.1-10.5$ & 44 & 12.8 & $9.2-16.4$ & 57 & 19.0 & $14.5-23.5$ & 61 & 21.3 & $16.4-26.1$ \\
\hline Total closures & 137 & & & 207 & & & 263 & & & 303 & & & 322 & & \\
\hline $\begin{array}{l}\text { Continuation with } \\
\text { device (net rate) }\end{array}$ & & 73.8 & & & 59.3 & & & 47.0 & & & 37.5 & & & 32.5 & \\
\hline Lost to follow-up & 69 & & & 86 & & & 99 & & & 108 & & & 110 & & \\
\hline
\end{tabular}

CI, 95\% Confidence intervals; GR, gross rate; IUD, intrauterine device; n, number; PID, pelvic inflammatory disease.

Table 4 Nova $T_{380^{\circledR}}$, Nova $T^{\circledR}$ and $C u T 380^{\circledR}$ : cumulative gross failure rates per 100 users at 5 years

\begin{tabular}{|c|c|c|c|c|c|c|c|c|}
\hline Study & Location & Device & $\mathrm{n}$ & Pregnancy & Expulsion & $\begin{array}{l}\text { Bleeding and } \\
\text { bleeding with pain }\end{array}$ & Pain & PID \\
\hline Present study & UK & Nova T380 ${ }^{\circledR}$ & 574 & 2 & 13 & 29.5 & 7.1 & 0.9 \\
\hline Andersson et al. $(1994)^{10}$ & Scandinavia, Hungary & Nova $T^{\circledR}$ & 937 & 5.9 & 6.7 & 20.9 & 5.8 & 2.2 \\
\hline Luukkainen et al. $(1986)^{11}$ & Finland & Nova $T^{\circledR}$ & 134 & 6.7 & 6 & 21.7 & & \\
\hline Sivin et al. $(1990)^{15}$ & US and five others & 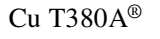 & 1121 & 1.4 & 7.4 & 23.3 & & \\
\hline
\end{tabular}

PID, Pelvic inflammatory disease.

Table 5 Bleeding problems leading to IUD removal at or before 5 years of use

\begin{tabular}{lrr}
\hline Reasons for removal & $\mathrm{n}$ & $\%$ \\
\hline Heavier periods/'bleeding' & 73 & 71 \\
Continuous or persistent vaginal loss & 21 & 20 \\
Irregular bleeding & 6 & 6 \\
Other $^{\mathrm{a}}$ & 3 & 3 \\
Total & 103 & 100 \\
\hline
\end{tabular}

a'Other' includes: heavy bleeding due to fibroid; bleeding and pain due to large polyp; pain.

included in the 24-month report ${ }^{2}$ was excluded after further information became available. One additional case that occurred at 3 months of use was reported after the end of the first 12 months and so was not included in the 12-month report. ${ }^{1}$ The IUD was removed in six cases though two of them were recorded as removed because of pain. Therefore, although ten women are reported with PID in Table 6, only four removals for PID are recorded in Table 3. Of the four all were treated successfully with antibiotics without admission to hospital. The relationship of PID to IUD use is uncertain and is discussed below.

Other reasons for IUD removal

A total of 61 women had the device removed at or before 5 years for various other reasons shown in Table 7. Accidental removal of the device by the subject was not included with expulsions, being distinguished by the fact that the women knew that the device had been removed and they were therefore fully aware that they were unprotected.

Table 6 Pelvic inflammatory disease (PID)

\begin{tabular}{|c|c|c|c|c|c|}
\hline Age (years) & $\begin{array}{l}\text { Duration of IUD } \\
\text { use (months) }\end{array}$ & Severity & $\begin{array}{l}\text { Investigation/ } \\
\text { treatment }\end{array}$ & IUD removed & Result \\
\hline 32 & 1 & Moderate & Augmentin, metronidazole & No & Cured \\
\hline 22 & 3 & - & Antibiotics, Canesten & No & Cured \\
\hline 37 & 4 & Moderate & Oxytetracycline & Yes & Cured \\
\hline 20 & 4 & Moderate & Two episodes, antibiotics & Yes, at second episode & Cured \\
\hline $21^{\mathrm{a}}$ & 6 & - & Treated as hospital outpatient & Yes & Cured \\
\hline 40 & 6.5 & Mild & Doxycycline, metronidazole & No & Cured \\
\hline 29 & 7 & Mild & Penicillin & Yes & Cured \\
\hline $29^{b}$ & 22 & Severe & Information not available & Yes (admitted to hospital) & Cured \\
\hline 32 & 31 & - & Antibiotics & No & Cured \\
\hline $28^{b}$ & 53 & Moderate & Doxycycline, metronidazole & Yes & Improved at last contact \\
\hline
\end{tabular}

a Diagnosis doubtful as little information available from genitourinary medicine (GUM) clinic which treated patient.

'Reason for removal recorded as 'pain'.

IUD, Intrauterine device. 
Table 7 Other reasons for IUD removal

\begin{tabular}{lrr}
\hline Reason for removal & $\mathrm{n}$ & $\%$ \\
\hline Sterilisation/partner vasectomy & 21 & 35 \\
Accidental removal by subject $^{\text {Investigator's decision }}{ }^{\mathrm{a}}$ & 9 & 15 \\
IUD no longer needed (partner death, end of relationship) $^{-}$ & 9 & 15 \\
Reason not known $_{\text {Subject prefers alternative method }}$ & 7 & 11 \\
Subject's request $^{\mathrm{b}}$ & 5 & 8 \\
Total & 3 & 5 \\
\end{tabular}

anvestigator's decision [actinomyces-like organisms (ALOs) on smear (5), lost threads, subject moving abroad, locum thought IUD too low, GP thought should be changed].

bSubject's request (loss of confidence in IUD, partner opposed, reason not known).

\section{Discussion}

Table 4 shows the published results of 5-year studies of the Nova $T^{\circledR}$ together with a 5 -year study of the $\mathrm{Cu} T 380 \mathrm{~A}^{\circledR}$. (The similar Gyne-T380S ${ }^{\circledR}$ was recently withdrawn from the UK market but replaced by the $\mathrm{Cu}$ T380 ${ }^{\circledR}$ also called the T-Safe $380 \mathrm{~A}^{\circledR}$.) Comparison with the $\mathrm{Cu}$ T380A ${ }^{\circledR}$ is relevant because it has been stated 'it is against this standard that we must judge other devices'. ${ }^{\text {? }}$

In our 12-month report we concluded that on the basis of 12 months' data 'increasing the surface area of copper did not seem to be associated with a reduced pregnancy rate'. We also stated that 'results beyond 12 months of use may in due course give additional information'. Indeed, the pregnancy rate in our study at 5 years is substantially lower that that of the two Nova $\mathrm{T}^{\circledR}$ studies; namely 2.0 (or 2.82 ITT) compared to $5.9^{10}$ and 6.7.11 Therefore although the present study was not a direct comparative study with the Nova $T^{\circledR}$, it lends weight to the notion that increasing the copper reduces the pregnancy rate.

The diagnosis of PID depended on the clinical judgement of the clinician involved, as laparoscopy was not used. The ten cases of PID could have been related to the IUD. However the frequency of PID has been shown in recent studies to be only minimally, if at all, related to the copper IUD. Of greater significance is exposure to sexually transmitted infection (STI) rather than the presence of an IUD. ${ }^{12}$ During the period of this study only some centres were carrying out screening and prophylactic measures for Chlamydia and other organisms. There appeared to be no increased risk of PID soon after fitting as has been reported elsewhere. ${ }^{13} \mathrm{~A}$ recent case-control study concluded that 'a previous use of a copper IUD is not associated with an increased risk of tubal occlusion among nulligravid women'. ${ }^{14}$

The removal rate for bleeding and bleeding with pain in the present study at 29.6 is higher than in the Nova $\mathrm{T}^{\circledR}$ studies (Table 4). There is no obvious explanation for the difference. Can the extra copper be blamed or are the UK women less tolerant of bleeding/pain?

The expulsion rate in the present study was also higher than in the Nova $\mathrm{T}^{\circledR}$ studies. The unexpectedly high expulsion rate is unlikely to be due to the increased copper or design of the device because the $\mathrm{Cu}$ T380A ${ }^{\circledR}$ study (Table 4) had an expulsion rate similar to the Nova $\mathrm{T}^{\circledR}$ studies.

It is noteworthy that a substantial majority of closures in all categories except 'other reasons' occurred during the first 2 years (Table 3 ).

\section{Conclusions}

At 5 years of use in the UK the Nova T380 ${ }^{\circledR}$ IUD has been shown to have a very low pregnancy rate. In the present study, increasing the surface area of copper was associated with a reduced pregnancy rate but this was not a direct comparative study with the Nova $\mathrm{T}^{\circledR}$. There was a higher than expected removal rate for pain/bleeding and a higher expulsion rate than in Nova $\mathrm{T}^{\circledR}$ studies. The removal for PID rate was lower than in Nova $T^{\circledR}$ studies. Other complications were similar to other published studies. The study shows that this device is a welcome addition to the copper-bearing IUDs available.

\section{Acknowledgements}

The Nova T380 ${ }^{\circledR}$ IUDs were supplied by Leiras Oy. Statistical analysis was by Ruth Preist, Hilary Hardwicke and Sarah Blacksell of the School of Postgraduate Medicine, University of Exeter.

\section{Statements on funding and competing interests}

Funding. The study was sponsored by Leiras Oy and Schering Health Care Limited.

Competing interests. None declared.

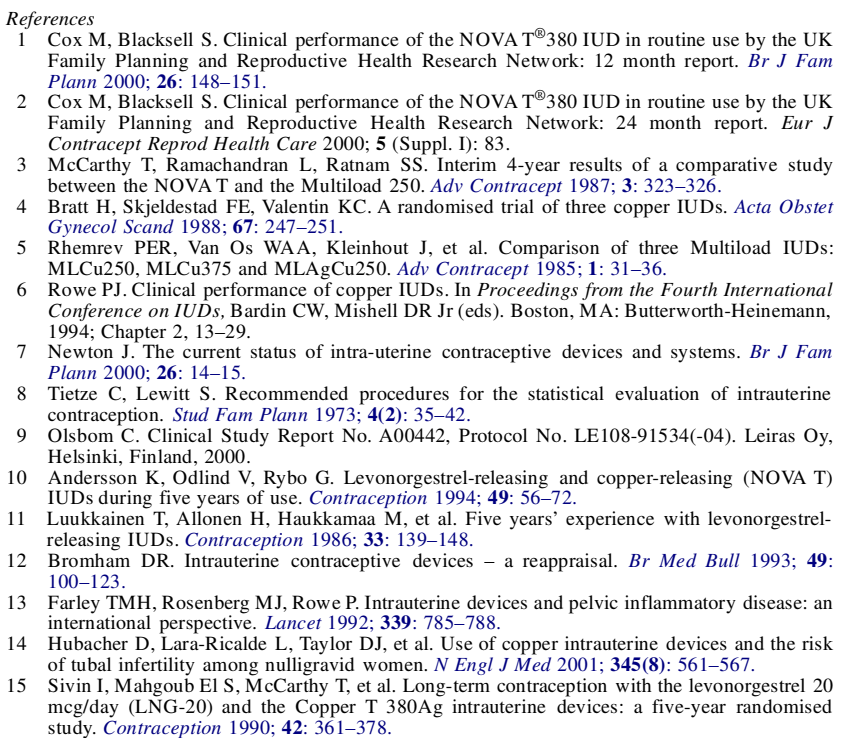

Cox M, Blacksell S. Clinical performance of the NOVA T ${ }^{\circledR} 380$ IUD in routine use by the UK

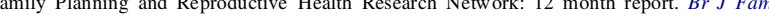
Cox M, Blacksell S. Clinical performance of the NOVA T ${ }^{\circledR} 380$ IUD in routine use by the UK Family Planning and Reproductive Health Research Network: 24 month report. Eur J (Suppl. I): 83.

ndomised trial of three copper IUDs. Acta Obstet :

MLCu250, MLCu375 and MLAgCu250. Adv Contracept 1985; 1:31-36.

Rowe PJ. Clinical performance of copper IUDs. In Proceedings from the Fourth International Confer , Allonen $\mathrm{H}$, Haukkamaa M, et al.

Br Med Bull 1993; 49:

eses an (a)

of tubal infertility among nulligravid women. $N$ Engl J Med 2001; 345(8):561-567.

$\mathrm{mcg} / \mathrm{day}$ (LNG-20) and the Copper T $380 \mathrm{Ag}$ intrauterine devices: a five-year randomised study. Contraception 1990; 42: 361-378. 